\title{
Stochastic Non-autonomous Lotka-Volterra Mutualism systems with Impulse Jump and Markov Switching
}

\author{
Fangxia Lu ${ }^{a}$, Zuomao Yan ${ }^{b}$ \\ Department of Mathematics, Hexi University, Gansu 734000, China \\ azhylfx@163.com, byanzuomao@163.com
}

\begin{abstract}
In this paper, we investigate a stochastic non-autonomous Lotka-Volterra mutualism with impulse jump and Markov switching, and two sufficient conditions for stochastic persistence are established
\end{abstract}

Keywords: Brownian motion; Mpulse jump; Markov switching; Generalized It formul; Stochastic permistence.

\section{Introduction}

The classical non-autonomous Lotka-Volterra mutualism system can be described as follows:

$$
\frac{d x_{i}(\mathrm{t})}{d t}=x_{i}(\mathrm{t})\left[r_{i}(\mathrm{t})-a_{i i}(\mathrm{t}) x_{i}(\mathrm{t})+\sum_{\substack{j=1 \\ j \neq i}}^{n} a_{i j}(\mathrm{t}) x_{j}(\mathrm{t})\right]
$$

Extensive literature concerned mutualism system (1) and its generalized forms.

The above mentioned papers are all deterministic models, which do not consider the effect of environmental noise for population system. In fact, population system is often subject to effect by environmental noise (see [1-3]). In paper [2-3], some result on the nonexplosion, boundedness and persistence for stochastic population systems have been developed. Particularly, Ji and Jiang in [3] studied nonautonomous two-species stochastic Lotka-Volterra mutualism model

$$
\left\{\begin{array}{l}
d x_{1}(t)=x_{1}(t)\left[\left(r_{1}(t)-a_{11}(t) x_{1}(t)+a_{12}(t) x_{2}(t)\right) d t+\sigma_{1}(t) d B_{1}(t)\right] \\
d x_{2}(t)=x_{2}(t)\left[\left(r_{2}(t)-a_{21}(t) x_{1}(t)-a_{22}(t) x_{2}(t)\right) d t+\sigma_{2}(t) d B_{2}(t)\right]
\end{array}\right.
$$

For the systems (2) and its generalized forms, many interesting results have been obtained.

On the other hand, the growth of species usually undergoes some discrete changes of relatively short time interval at some fixed times, such as drought, flooding, earthquake, planting, harvesting etc. From point view of mathematic, the sudden changes could be described by impulses. In this case, impulsive effects should be taken into account system (2). Some results (see [4-7]) have been proposed for stochastic systems with impulsive effects. Up to now, little research has been done about stochastic non-autonomous Lotka-Volterra mutualism with both impulse effects and Markov switching. Motivated by these, in this paper we consider the following non-autonomous two-species stochastic Lotka-Volterra mutualism with impulse jump and Markov switching system:

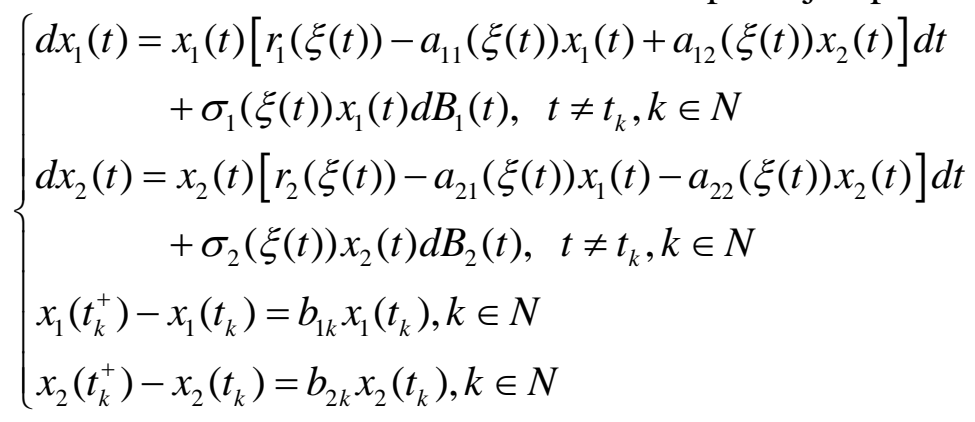

Where $N$ denotes the set of positive integers, $0<t_{1}<t_{2} \cdots, \lim _{k \rightarrow+\infty} t_{k}=+\infty$. On count of biological meanings we impose the additional restrictions on systems (3), 
$b_{i k}>-1, i=1,2, \mathrm{~L} n, k \in N$.

When $b_{i k}>0$, the impulsive effects denote planting, while $b_{i k}<0$ represent harvesting [14]. $\xi(t)$ is Markov switching take values in $S=\{1,2, \mathrm{~L}, \mathrm{~N}\}$, switching from one mode to the others according to movement of the Markov chain $\xi(t) . B_{i}(t), i=1,2$ are mutually independent one dimensional standard Brownian motions with $B_{i}(0)=0$.

\section{Stochastic persistence}

Let $R_{+}^{2}$ denote positive cone of $R^{2}$. For $x \in R^{2},|x|=\left|x_{1}\right|+\left|x_{2}\right|$. Moreover, we assume that $\inf _{t \geq 0} a_{i i}(\xi(t))>0$ for all $i=1,2$ and $a_{i j}(\xi(t))>0$ for all $i=1,2$ with $j \neq i$.

Studying a population system, we pay more attention on whether the system is persistent. In this section, we first show that the solution is stochastic permanence. Before give the main theorems, we first give some famous concepts, lemmas and assumptions (see e.g. [7]).

Definition 1 system (3) is sad to be stochastically permanent if for every $\varepsilon \in(0,1)$, there are constants $M=M(\varepsilon), N=N(\varepsilon)$ such that

$\liminf _{t \rightarrow+\infty}\{x(t) \geq M\} \geq 1-\varepsilon, \liminf _{t \rightarrow+\infty}\{x(t) \leq N\} \geq 1-\varepsilon$.

Assumption 1 For some $u \in S, q_{i u}>0(\forall i \neq u)$.

Assumption $2 \sum_{k=1}^{n} \pi_{u} \hat{\beta}(u)>0, \hat{\beta}(u)=\stackrel{\vee}{b}(u)-\frac{1}{2} \hat{\sigma}^{2}(u), \stackrel{\vee}{b}(u)=\min _{1 \leq i, j \leq 2} b_{i j}(u), u \in S$.

Assumption $3 \hat{\beta}(u)>0, u \in S$.

Lemma 1 (see e.g. [4]). Asumptions 1 and 2 imply that there exists a constant $\theta>0$ such that the matrix $A(\theta)$ is a nonsingular M-matrix, where

$$
A(\theta)=\operatorname{diag}\left(\xi_{1}(\theta), \xi_{2}(\theta), \cdots, \xi_{n}(\theta)\right)-\Gamma, \quad \xi_{u}(\theta)=\theta \beta(u)-\frac{1}{2} \theta^{2} \sigma^{2}(u), \forall u \in S .
$$

Lemma 2 Asumption 3 imply that there exists a constant $\theta>0$ such that the matrix $A(\theta)$ is a nonsingular M-matrix.

Assumption 4 There exist two constants $m>0$ and $M>0$ such that $m \leq \prod_{0<t_{k}<t}\left(1+b_{i k}\right) y_{i}(t) \leq \mathrm{M}_{\text {for }}$ all $t>0, i=1,2$.

Theorem 2 If assumption (1), (2) and (4) hold, then system (3) is stochastically permanent.

Proof. Firstly, let us show that for given $\varepsilon \in(0,1)$, there exists a positive constant $M$ such that $\liminf _{t \rightarrow+\infty}\{x(t) \geq M\} \geq 1-\varepsilon$. Applying the generalized It $\hat{o}$ formula, we compute

$$
\begin{aligned}
d\left(\frac{1}{y_{1}}\right)= & -\frac{1}{y_{1}{ }^{2}} d y_{1}+\frac{1}{y_{1}{ }^{3}}\left(\mathrm{~d} y_{1} \cdot d y_{1}\right) \\
= & -\frac{1}{y_{1}}\left[r_{1}(\xi)-a_{11}(\xi) \prod_{0<t_{k}<t}\left(1+b_{1 k}\right) y_{1}+a_{12}(\xi) \prod_{0<t_{k}<t}\left(1+b_{2 k}\right) y_{2}\right] d t \\
& +\frac{1}{y_{1}} \sigma_{1}{ }^{2}(\xi) y_{1} d t-\frac{1}{y_{1}} \sigma_{1}(\xi) d B_{1}(t) . \\
d\left(\frac{1}{y_{2}}\right)= & -\frac{1}{y_{2}{ }^{2}} d y_{1}+\frac{1}{y_{2}{ }^{3}}\left(\mathrm{~d} y_{1} \cdot d y_{1}\right)
\end{aligned}
$$




$$
\begin{aligned}
= & -\frac{1}{y_{2}}\left[r_{2}(\xi)+a_{21}(\xi) \prod_{0<t_{k}<t}\left(1+b_{1 k}\right) y_{1}-a_{22}(\xi) \prod_{0<t_{k}<t}\left(1+b_{2 k}\right) y_{2}\right] d t \\
& +\frac{1}{y_{2}} \sigma_{2}^{2}(\xi) d t-\frac{1}{y_{2}} \sigma_{2}(\xi) d B_{2}(t) .
\end{aligned}
$$

For $\theta$ given in lemma 3, by lemma 2, there is a vector $\vec{q}=\left(\mathrm{q}_{1}, \mathrm{q}_{2}, \cdots \mathrm{q}_{N}\right)^{T} \square 0$ such that $A(\theta) \vec{q} \square 0$, namely

$$
q_{u}\left(\theta \hat{\beta}(u)-\frac{1}{2} \theta^{2} \tilde{\sigma}^{2}(u)\right)-\sum_{l=1}^{N} \gamma_{u l} q_{l} \text { for all } 1 \leq u \leq N .
$$

Define $V_{1}(y, u)=q_{u}\left(1+y_{1}^{-1}\right)^{\theta}+q_{u}\left(1+y_{2}^{-1}\right)^{\theta}$.Then, by the generalized It $\hat{o}$ formula, we have $E V_{1}(y(t), \xi(t))=V_{1}(y(0), \xi(0))+E \int_{0}^{t} L V_{1}(y(s), \xi(s)) d s$,

Where

$$
\begin{aligned}
& L V_{1}(y, u)=q_{u} \theta\left(1+\frac{1}{y_{1}}\right)^{\theta-1} d \frac{1}{y_{1}}+\frac{1}{2} q_{u} \theta(\theta-1)\left(1+\frac{1}{y_{1}}\right)^{\theta-2} d\left(\frac{1}{y_{1}}\right)^{2} \\
& +q_{u} \theta\left(1+\frac{1}{y_{2}}\right)^{\theta-1} d \frac{1}{y_{2}}+\frac{1}{2} q_{u} \theta(\theta-1)\left(1+\frac{1}{y_{2}}\right)^{\theta-2} d\left(\frac{1}{y_{2}}\right)^{2}+\sum_{l=1}^{N} \gamma_{u l} V_{1}(y, l) q_{l} \\
& =q_{u} \theta\left(1+\frac{1}{y_{1}}\right)^{\theta-2}\left\{( 1 + \frac { 1 } { y _ { 1 } } ) \left[-\frac{1}{y_{1}}\left(r_{1}(\xi)-a_{11}(\xi) \prod_{0<t_{k}<t}\left(1+b_{1 k}\right) y_{1}+a_{12}(\xi) \prod_{0<t_{k}<t}\left(1+b_{2 k}\right) y_{2}\right)\right.\right. \\
& \left.\left.+\frac{1}{y_{1}} \sigma_{1}^{2}(\xi)\right]+\frac{1}{2}(\theta-1)\left(\frac{\sigma_{1}(\xi)}{y_{1}}\right)^{2}\right\}+q_{u} \theta\left(1+\frac{1}{y_{2}}\right)^{\theta-2}\left\{( 1 + \frac { 1 } { y _ { 2 } } ) \left[-\frac{1}{y_{2}}\left(r_{2}(\xi)+a_{21}(\xi) \prod_{0<t_{k}<t}\left(1+b_{1 k}\right) y_{1}\right.\right.\right. \\
& \left.\left.\left.-a_{22}(\xi) \prod_{0<t_{k}<t}\left(1+b_{2 k}\right) y_{2}\right)+\frac{1}{y_{2}} \sigma_{2}^{2}(\xi)\right]+\frac{1}{2}(\theta-1)\left(\frac{\sigma_{2}(\xi)}{y_{2}}\right)^{2}\right\}+\left(1+\frac{1}{y_{1}}\right)^{\theta} \sum_{l=1}^{N} \gamma_{u l} q_{l}+\left(1+\frac{1}{y_{2}}\right)^{\theta} \sum_{l=1}^{N} \gamma_{u l} q_{l} \\
& =\left(1+\frac{1}{y_{1}}\right)^{\theta-2}\left\{-\frac{1}{y_{1}^{2}} q_{u} \theta\left[r_{1}(\xi)-\sigma_{1}^{2}(\xi)-\frac{1}{2}(\theta-1) \sigma_{1}^{2}(\xi)\right]+\frac{1}{y_{1}} q_{u} \theta\left[-r_{1}(\xi)+\sigma_{1}^{2}(\xi)+a_{11}(\xi) \prod_{0<t_{k}<t}\left(1+b_{1 k}\right)\right]\right. \\
& \left.-\frac{y_{2}}{y_{1}}\left[q_{u} \theta\left(a_{12}(\xi) \prod_{0<t_{k}<t}\left(1+b_{2 k}\right)+\frac{a_{12}(\xi) \prod_{0<t_{k}<t}\left(1+b_{2 k}\right)}{y_{1}}\right)\right]+a_{11}(\xi) \prod_{0<t_{k}<t}\left(1+b_{1 k}\right)+\left(1+\frac{1}{y_{1}}\right)^{\theta} \sum_{l=1}^{N} \gamma_{u l} q_{l}\right\} \\
& +\left(1+\frac{1}{y_{2}}\right)^{\theta-2}\left\{-\frac{1}{y_{2}^{2}} q_{u} \theta\left[r_{2}(\xi)-\sigma_{2}^{2}(\xi)-\frac{1}{2}(\theta-1) \sigma_{2}^{2}(\xi)\right]+\frac{1}{y_{2}} q_{u} \theta\left[-r_{2}(\xi)+\sigma_{2}^{2}(\xi)\right.\right. \\
& \left.+a_{22}(\xi) \prod_{0<t_{k}<t}\left(1+b_{2 k}\right)\right]-\frac{y_{1}}{y_{2}}\left[q_{u} \theta\left(a_{21}(\xi) \prod_{0<t_{k}<t}\left(1+b_{1 k}\right)+\frac{a_{21}(\xi) \prod_{0<t_{k}<t}\left(1+b_{1 k}\right)}{y_{2}}\right)\right] \\
& +a_{22}(\xi) \prod_{0<t_{k}<t}\left(1+b_{2 k}\right)+\left(1+\frac{1}{y_{2}}\right)^{\theta} \sum_{l=1}^{N} \gamma_{u l} q_{l} \\
& =\left(1+\frac{1}{y_{1}}\right)^{\theta-2}\left\{-\frac{1}{y_{1}^{2}}\left[q_{u}\left(\theta r_{1}(u)-\frac{1}{2} \theta \sigma_{1}^{2}(u)-\frac{1}{2} \theta^{2} \sigma_{1}^{2}(u)\right)-\sum_{l=1}^{N} \gamma_{u l} q_{l}\right]+\frac{1}{y_{1}}\left[q _ { u } \theta \left(a_{11}(u) \prod_{0<t_{k}<t}\left(1+b_{1 k}\right)\right.\right.\right. \\
& \left.\left.-r_{1}(u)+\sigma_{1}^{2}(u)\right)+2 \sum_{l=1}^{N} \gamma_{u l} q_{l}\right]-\frac{y_{2}}{y_{1}}\left[q_{u} \theta\left(a_{12}(u) \prod_{0<t_{k}<t}\left(1+b_{2 k}\right)+\frac{a_{12}(u) \prod_{0<t_{k}<t}\left(1+b_{2 k}\right)}{y_{1}}\right)\right] \\
& \left.+\left[q_{u} \theta a_{11}(u) \prod_{0<t_{k}<t}\left(1+b_{1 k}\right)+\sum_{l=1}^{N} \gamma_{u l} q_{l}\right]\right\}+\left(1+\frac{1}{y_{2}}\right)^{\theta-2}\left\{-\frac{1}{y_{2}^{2}}\left[q_{u}\left(\theta r_{2}(u)-\frac{1}{2} \theta \sigma_{2}^{2}(u)-\frac{1}{2} \theta^{2} \sigma_{2}^{2}(u)\right)\right.\right. \\
& \left.-\sum_{l=1}^{N} \gamma_{u l} q_{l}\right]+\frac{1}{y_{2}}\left[q_{u} \theta\left(a_{22}(u) \prod_{0<t_{k}<t}\left(1+b_{2 k}\right)-r_{2}(u)+\sigma_{2}^{2}(u)\right)+2 \sum_{l=1}^{N} \gamma_{u l} q_{l}\right]-\frac{y_{1}}{y_{2}}\left[q _ { u } \theta \left(a_{21}(u) \prod_{0<t_{k}<t}\left(1+b_{1 k}\right)\right.\right.
\end{aligned}
$$




$$
\left.\left.\left.+\frac{a_{21}(u) \prod_{0<t_{k}<t}\left(1+b_{1 k}\right)}{y_{2}}\right)\right]+\left[q_{u} \theta a_{22}(u) \prod_{0<t_{k}<t}\left(1+b_{2 k}\right)+\sum_{l=1}^{N} \gamma_{u l} q_{l}\right]\right\} .
$$

Now, choose $\eta>0$ sufficiently small such that it satisfies $q_{u}\left(\theta r_{i}(u)-\frac{1}{2} \theta^{2} \sigma_{i}(u)\right)-\sum_{l=1}^{N} \gamma_{u l} q_{l}-\eta q_{u}>0$ for all $1 \leq u \leq N, i=1,2$.

Define $V_{2}(y, u)=e^{\eta t} V_{1}(y, u)=e^{\eta t} q_{u}\left(1+y_{1}^{-1}\right)^{\theta}+e^{\eta t} q_{u}\left(1+y_{2}^{-1}\right)^{\theta}$. Then, by the generalized It $\hat{o}$ formula

$$
E V_{2}(y(t), \xi(t))=V_{2}(y(0), \xi(0))+E \int_{0}^{t} L V_{2}(y(s), \xi(s)) d s,
$$

Where

$$
\begin{aligned}
L & V_{2}(y, u)=\eta e^{\eta t} V_{1}(y, u)+e^{\eta t} L V_{1}(y, u) \\
= & e^{\eta t}\left(1+y_{1}^{-1}\right)^{\theta-2}\left\{-\frac{1}{y_{1}^{2}}\left[q_{u} \theta r_{1}(u)-\frac{1}{2} q_{u} \theta \sigma_{1}^{2}(u)-\sum_{l=1}^{N} \gamma_{u l} q_{l}-\eta q_{u}\right]+\frac{1}{y_{1}}\left[q_{u} \theta a_{11}(u) \prod_{0<t_{k}<t}\left(1+b_{1 k}\right)\right.\right. \\
& \left.-q_{u} \theta r_{1}(u)+q_{u} \theta \sigma_{1}^{2}(u)+2 \sum_{l=1}^{N} \gamma_{u l} q_{l}+2 \eta q_{u}\right]-\frac{y_{2}}{y_{1}}\left[q_{u} \theta a_{12}(u) \prod_{0<t_{k}<t}\left(1+b_{2 k}\right)+\frac{q_{u} \theta a_{12}(u) \prod_{0<t_{k}<t}\left(1+b_{2 k}\right)}{y_{1}}\right] \\
& +\left[q_{u} \theta a_{11}(u) \prod_{0<t_{k}<t}\left(1+b_{1 k}\right)+\sum_{l=1}^{N} \gamma_{u l} q_{l}+\eta q_{u}\right]+e^{\eta t}\left(1+y_{2}^{-1}\right)^{\theta-2}\left\{-\frac{1}{y_{2}^{2}}\left[q_{u} \theta r_{2}(u)-\frac{1}{2} q_{u} \theta \sigma_{2}^{2}(u)-\sum_{l=1}^{N} \gamma_{u l} q_{l}-\eta q_{u}\right]\right. \\
& +\frac{1}{y_{2}}\left[q_{u} \theta a_{22}(u) \prod_{0<t_{k}<t}\left(1+b_{2 k}\right)-q_{u} \theta r_{2}(u)+q_{u} \theta \sigma_{2}^{2}(u)+2 \sum_{l=1}^{N} \gamma_{u l} q_{l}+2 \eta q_{u}\right]-\frac{y_{1}}{y_{2}}\left[q_{u} \theta a_{21}(u) \prod_{0<t_{k}<t}\left(1+b_{1 k}\right)\right. \\
& \left.+\frac{q_{u} \theta a_{21}(u) \prod_{0<t_{k}<t}\left(1+b_{1 k}\right)}{y_{2}}\right]+\left[q_{u} \theta a_{22}(u) \prod_{0<t_{k}<t}\left(1+b_{2 k}\right)+\sum_{l=1}^{N} \gamma_{u l} q_{l}+\eta q_{u}\right] \\
\leq & e^{\eta t}\left(1+y_{1}^{-1}\right)^{\theta-2}\left\{-\frac{1}{y_{1}^{2}}\left[q_{u} \theta r_{1}(u)-\frac{1}{2} q_{u} \theta \sigma_{1}^{2}(u)-\sum_{l=1}^{N} \gamma_{u l} q_{l}-\eta q_{u}\right]+\frac{1}{y_{1}}\left[q_{u} \theta a_{11}(u) M\right.\right. \\
& \left.\left.-q_{u} \theta r_{1}(u)+q_{u} \theta \sigma_{1}^{2}(u)+2 \sum_{l=1}^{N} \gamma_{u l} q_{l}+2 \eta q_{u}\right]+\left[q_{u} \theta a_{11}(u) M+\sum_{l=1}^{N} \gamma_{u l} q_{l}+\eta q_{u}\right]\right\} \\
& +e^{\eta t}\left(1+y_{2}^{-1}\right)^{\theta-2}\left\{-\frac{1}{y_{2}^{2}}\left[q_{u} \theta r_{2}(u)-\frac{1}{2} q_{u} \theta \sigma_{2}^{2}(u)-\sum_{l=1}^{N} \gamma_{u l} q_{l}-\eta q_{u}\right]+\frac{1}{y_{2}}\left[q_{u} \theta a_{22}(u) M\right.\right. \\
& \left.\left.-q_{u} \theta r_{2}(u)+q_{u} \theta \sigma_{2}^{2}(u)+2 \sum_{l=1}^{N} \gamma_{u l} q_{l}+2 \eta q_{u}\right]+\left[q_{u} \theta a_{22}(u) M+\sum_{l=1}^{N} \gamma_{u l} q_{l}+\eta q_{u}\right]\right\} \\
= & e^{\eta t} H(y, u),
\end{aligned}
$$

where

$$
\begin{aligned}
& H(y, u)=\left(1+y_{1}^{-1}\right)^{\theta-2}\left\{-\frac{1}{y_{1}^{2}}\left[q_{u} \theta r_{1}(u)-\frac{1}{2} q_{u} \theta \sigma_{1}^{2}(u)-\sum_{l=1}^{N} \gamma_{u l} q_{l}-\eta q_{u}\right]+\frac{1}{y_{1}}\left[q_{u} \theta a_{11}(u) M\right.\right. \\
&\left.\left.-q_{u} \theta r_{1}(u)+q_{u} \theta \sigma_{1}^{2}(u)+2 \sum_{l=1}^{N} \gamma_{u l} q_{l}+2 \eta q_{u}\right]+\left[q_{u} \theta a_{11}(u) M+\sum_{l=1}^{N} \gamma_{u l} q_{l}+\eta q_{u}\right]\right\} \\
&+\left(1+y_{2}^{-1}\right)^{\theta-2}\left\{-\frac{1}{y_{2}^{2}}\left[q_{u} \theta r_{2}(u)-\frac{1}{2} q_{u} \theta \sigma_{2}^{2}(u)-\sum_{l=1}^{N} \gamma_{u l} q_{l}-\eta q_{u}\right]+\frac{1}{y_{2}}\left[q_{u} \theta a_{22}(u) M\right.\right. \\
&\left.\left.-q_{u} \theta r_{2}(u)+q_{u} \theta \sigma_{2}^{2}(u)+2 \sum_{l=1}^{N} \gamma_{u l} q_{l}+2 \eta q_{u}\right]+\left[q_{u} \theta a_{22}(u) M+\sum_{l=1}^{N} \gamma_{u l} q_{l}+\eta q_{u}\right]\right\} .
\end{aligned}
$$

By the definition of $\eta, H(y, u)$ is upper bounded in $R_{+} \times S$, This implies 
$\left.\stackrel{q}{ } E^{\eta t}\left(1+y_{1}^{-1}\right)^{\theta}+e^{\eta t}\left(1+y_{2}^{-1}\right)^{\theta}\right] \leq \hat{q}\left(1+y_{1}^{-1}(0)\right)^{\theta}+\hat{q}\left(1+y_{2}^{-1}(0)\right)^{\theta}+\frac{H_{1}\left(e^{\eta t}-1\right)}{\eta}$.

That is to say

$\limsup _{t \rightarrow+\infty} E\left[y_{1}^{-\theta}(t)\right] \leq \limsup _{t \rightarrow+\infty} E\left[\left(1+y_{1}^{-1}(t)\right)^{\theta}+\left(1+y_{2}^{-1}(t)\right)^{\theta}\right] \leq \frac{H_{1}}{\eta q}=: H_{2}$

Consequently

$\limsup _{t \rightarrow+\infty} E\left[x_{1}^{-\theta}(t)\right] \leq \limsup _{t \rightarrow+\infty} E\left[\left(\prod_{0<t_{k}<t}\left(1+b_{1 k}\right) y_{1}\right)^{-\theta}\right] \leq m^{-\theta} H_{2}=: H_{3}$

$\limsup _{t \rightarrow+\infty} E\left[x_{2}^{-\theta}(t)\right] \leq \limsup _{t \rightarrow+\infty} E\left[\left(\prod_{0<t_{k}<t}\left(1+b_{2 k}\right) y_{2}\right)^{-\theta}\right] \leq H_{3}$.

For any given $\varepsilon>0$, let $M=\left(\frac{\varepsilon}{\mathrm{H}_{2}}\right)^{\frac{1}{\theta}}$, by the Chebyshev inequality, we can obtain that

$P\left\{x_{i}(t)<M\right\}=P\left\{x_{i}^{-\theta}(t)<M^{-\theta}\right\} \leq M^{\theta} E\left[x_{i}^{-\theta}(t)\right] ; i=1,2$.

Then,

$\limsup P\left\{x_{i}(t)<M\right\} \leq M H_{2}=\varepsilon$.

Consequently

$\liminf _{t \rightarrow+\infty} P\left\{x_{i}(t) \geq M\right\} \geq 1-\varepsilon, \quad i=1,2$.

Next we prove that for any given $\varepsilon>0$, there exists a positive constant $N$ such that $\liminf _{t \rightarrow+\infty} P\left\{x_{i}(t) \leq N\right\} \geq 1-\varepsilon, \quad i=1,2$.

If $a_{11}(\xi) a_{22}(\xi)-a_{12}(\xi) a_{21}(\xi)>0, \xi \in S$, then there exist positive numbers $c_{1}(\xi)$ and $c_{2}(\xi)$ for each $\xi \in S$ such that

$-\lambda:=\max _{k \in S}\left\{\lambda_{\max }^{+}\left(C(\xi) \mathrm{A}(\xi)+\mathrm{A}^{T}(\xi) \mathrm{C}(\xi)\right)\right\}<0$

Where $A(\xi)=\left(\begin{array}{cc}-a_{11}(\xi) \prod_{0<t_{k}<t}\left(1+b_{1 k}\right) & a_{12}(\xi) \prod_{0<t_{k}<t}\left(1+b_{2 k}\right) \\ a_{21}(\xi) \prod_{0<t_{k}<t}\left(1+b_{1 k}\right) & -a_{22} \prod_{0<t_{k}<t}\left(1+b_{2 k}\right)\end{array}\right), C(\xi)=\left(\begin{array}{cc}\mathrm{c}_{1}(\xi) & 0 \\ 0 & \mathrm{c}_{2}(\xi)\end{array}\right)$.

Define $V(y)=h_{1} y_{1}+h_{2} y_{2}$ for $y \in R_{+}^{2}$, where $h_{1}>0$ and $h_{2}>0$. Applying the generalized It $\hat{o}$ formula yields

$d V(y)=L V(y)+h_{1} \sigma_{1}(\xi) y_{1} d B_{1}(t)+h_{2} \sigma_{2}(\xi) y_{2} d B_{2}(t)$

Where

$$
\begin{aligned}
L V(y)= & h_{1} y_{1}\left[r_{1}(\xi)-a_{11}(\xi) \prod_{0<t_{k}<t}\left(1+b_{1 k}\right) y_{1}+a_{12}(\xi) \prod_{0<t_{k}<t}\left(1+b_{2 k}\right) y_{2}\right] \\
& +h_{2} y_{2}\left[r_{2}(\xi)+a_{21}(\xi) \prod_{0<t_{k}<t}\left(1+b_{1 k}\right) y_{1}-a_{22}(\xi) \prod_{0<t_{k}<t}\left(1+b_{2 k}\right) y_{2}\right] \\
= & h_{1} r_{1}(\xi) y_{1}+h_{2} r_{2}(\xi) y_{2}+\frac{1}{2} y^{T}\left(\mathrm{C}(\xi) \mathrm{A}+\mathrm{A}^{T} \mathrm{C}(\xi) \mathrm{y}\right. \\
\leq & h_{1} r_{1}(\xi) y_{1}+h_{2} r_{2}(\xi) y_{2}-\frac{\lambda}{2}\left(y_{1}^{2}+y_{2}^{2}\right)
\end{aligned}
$$

(4) implies that there exists a constant $H_{4}>0$ such that $L V(y)<H_{4}$. Making use of the generalized It $\hat{o}$ formula again leads to 


$$
\begin{aligned}
d\left[e^{t} V(y)\right]= & e^{t}\left(h_{1} y_{1}+h_{2} y_{2}\right) d t+e^{t}(L V(y)) d t+e^{t}\left[h_{1} \sigma_{1}(\xi) y_{1} d B_{1}(t)+h_{2} \sigma_{2}(\xi) y_{2} d B_{2}(t)\right] \\
\leq & e^{t}\left[h_{1} y_{1}+h_{2} y_{2}+h_{1} r_{1}(\xi) y_{1}+h_{2} r_{2}(\xi) y_{2}-\frac{\lambda}{2}\left(y_{1}^{2}+y_{2}^{2}\right)\right] \mathrm{dt}+e^{t}\left[h_{1} \sigma_{1}(\xi) y_{1} d B_{1}(t)+h_{2} \sigma_{2}(\xi) y_{2} d B_{2}(t)\right] \\
\leq & H_{4} e^{t} d t+e^{t}\left[h_{1} \sigma_{1}(\xi) y_{1} d B_{1}(t)+h_{2} \sigma_{2}(\xi) y_{2} d B_{2}(t)\right] . \\
d\left[e^{t} V(y)\right] & =e^{t}\left(h_{1} y_{1}+h_{2} y_{2}\right) d t+e^{t}(L V(y)) d t+e^{t}\left[h_{1} \sigma_{1}(\xi) y_{1} d B_{1}(t)+h_{2} \sigma_{2}(\xi) y_{2} d B_{2}(t)\right] \\
& \leq e^{t}\left[h_{1} y_{1}+h_{2} y_{2}+h_{1} r_{1}(\xi) y_{1}+h_{2} r_{2}(\xi) y_{2}-\frac{\lambda}{2}\left(y_{1}^{2}+y_{2}^{2}\right)\right] \mathrm{dt}+e^{t}\left[h_{1} \sigma_{1}(\xi) y_{1} d B_{1}(t)+h_{2} \sigma_{2}(\xi) y_{2} d B_{2}(t)\right] \\
& \leq H_{4} e^{t} d t+e^{t}\left[h_{1} \sigma_{1}(\xi) y_{1} d B_{1}(t)+h_{2} \sigma_{2}(\xi) y_{2} d B_{2}(t)\right] .
\end{aligned}
$$

Integrating from 0 to $t$ and taking expectations on both, we get

$e^{t} E V(y(\mathrm{t}))=V(y(0))+H_{4}\left(e^{t}-1\right)=h_{1} y_{10}+h_{2} y_{20}+H_{4}\left(e^{t}-1\right)$.

That is to say, $\limsup E[V(y(\mathrm{t}))] \leq H_{4}$. On the other hand, it is easy to see

that

$$
y_{1}+y_{2} \leq \frac{V(y(t))}{\min \left\{h_{1}, h_{2}\right\}}
$$

Therefore,

$\limsup _{t \rightarrow+\infty} E\left[x_{1}(t)\right] \leq \limsup _{t \rightarrow+\infty} E\left[\prod_{0<t_{k}<t}\left(1+b_{1 k}\right) y_{1}(t)\right] \leq M \limsup _{t \rightarrow+\infty} E y_{1}(t) \leq M \limsup _{t \rightarrow+\infty} \frac{V(y(t))}{\min \left\{h_{1}, h_{2}\right\}}$

$\leq \frac{M H_{4}}{\min \left\{h_{1}, h_{2}\right\}}=: H_{5}$, i.e. $\limsup _{t \rightarrow+\infty} E\left[x_{1}(t)\right] \leq H_{5}$.Thus for any given $\varepsilon>0$, Then by virtue of

Chebyshev inequality, we can derive that

$$
P\left\{x_{i}(t)>N\right\} \leq \frac{E\left[x_{i}(t)\right]}{N}=\frac{\varepsilon}{H_{5}} E\left[x_{i}(t)\right], i=1,2 .
$$

That is to say

$$
\limsup _{t \rightarrow+\infty} P\left\{x_{i}(t)>N\right\} \leq \limsup _{t \rightarrow+\infty} \frac{E\left[x_{i}(t)\right]}{N}=\limsup _{t \rightarrow+\infty} \frac{\varepsilon}{H_{5}} E\left[x_{i}(t)\right] \leq \varepsilon, i=1,2 .
$$

Theorem 2 If assumption (3) and (4) hold, then system (3) is stochastically permanent.

The proof of Theorem 2 is very similar to the proof Theorem 1 we omit the details here to avoid repetition.

\section{Summary}

This paper is concerned with stochastic persistence of Stochastic Non-autonomous Lotka-Volterra Mutualism systems with Impulse Jump and Markov Switching. Two sufficient conditions for stochastic persistence are obtained. If impulse is bounded and Assumption 2-4 hold, Note that both the impulse and color noise have no impact on stochastic persistence of the population system (3). Some more interesting result deserve further investigation, we will attempt to investigate persistence in mean, weak-persistence, non-persistence, extinction and global attractivity of the system (3) at the next stage.

\section{Acknowledgements}

The authors would like to thank the reviewer for the insightful comments. Addressing them has improved the quality of the paper. This research was partially supported by youth teacher research fund of Hexi University (No. QN2015-01).

\section{References}

[1] X. Mao, G. Marion, E. Renshaw, Environmental noise suppresses explosion in population dynamics. Stochastic Process Appl. Vol.97 (2002) No.2, p95-110. 
[2] X. Li, D. Jiang, X. Mao. Population dynamical behavior of Lotka-Volterra system under regime switching. Comput Appl Math. Vol.232(2009) No.6, p427-448

[3] C. Y. Ji, D. Q. Jiang, H. Liu, etal, Existence, uniqueness and ergodicity of positive solution of mutualism system with stochastic perturbation. Mathematical Problems in Engineering. Vol.2010 (2010) 10-25.

[4] M. Liu, K. Wang. On a stochastic logistic equation with impulsive perturbationsJ. Comput. Math. Appl. Vol.63 (2012) No.5, p871-886.

[5] M. Liu, K. Wang. Dynamics and simulations of a logistic Model with impulsive perturbations in a random environment. Math. Comput. Simulation. Vol.92(2013) No.4, p53-75

[6] M. Liu, K. Wang. Asymptotic behavior of a stochastic autonomous Lotka-Volterra competitive system with impulsive perturbations. Math. Comput. Modelling. Vol.271 (2015) No.11, p418-428.

[7] R. H. Wu, X. L. Zou, etal. Asymptotic properties of a stochastic Lotka-Volterra cooperative system with impulsive perturbations, Nonlinear Dyn. Vol.77 (2014) No.3, p807-817. 